\title{
Normalization with genes encoding ribosomal proteins but not GAPDH provides an accurate quantification of gene expressions in neuronal differentiation of $\mathrm{PC} 12$ cells
}

\author{
Lihan Zhou ${ }^{1,2}$, Qing-En Lim¹, Guoqiang Wan ${ }^{1,2}$, Heng-Phon Too ${ }^{1,2^{*}}$
}

\begin{abstract}
Background: Gene regulation at transcript level can provide a good indication of the complex signaling mechanisms underlying physiological and pathological processes. Transcriptomic methods such as microarray and quantitative realtime PCR require stable reference genes for accurate normalization of gene expression. Some but not all studies have shown that housekeeping genes (HGKs), $\beta$-actin (ACTB) and glyceraldehyde-3-phosphate dehydrogenase (GAPDH), which are routinely used for normalization, may vary significantly depending on the cell/tissue type and experimental conditions. It is currently unclear if these genes are stably expressed in cells undergoing drastic morphological changes during neuronal differentiation. Recent meta-analysis of microarray datasets showed that some but not all of the ribosomal protein genes are stably expressed. To test the hypothesis that some ribosomal protein genes can serve as reference genes for neuronal differentiation, a genome-wide analysis was performed and putative reference genes were identified based on stability of expressions. The stabilities of these potential reference genes were then analyzed by reverse transcription quantitative real-time PCR in six differentiation conditions.

Results: Twenty stably expressed genes, including thirteen ribosomal protein genes, were selected from microarray analysis of the gene expression profiles of GDNF and NGF induced differentiation of PC12 cells. The expression levels of these candidate genes as well as ACTB and GAPDH were further analyzed by reverse transcription quantitative real-time PCR in PC12 cells differentiated with a variety of stimuli including NGF, GDNF, Forskolin, KCl and ROCK inhibitor, Y27632. The performances of these candidate genes as stable reference genes were evaluated with two independent statistical approaches, geNorm and NormFinder.

Conclusions: The ribosomal protein genes, RPL19 and RPL29, were identified as suitable reference genes during neuronal differentiation of PC12 cells, regardless of the type of differentiation conditions. The combination of these two novel reference genes, but not the commonly used HKG, GAPDH, allows robust and accurate normalization of differentially expressed genes during PC12 differentiation.
\end{abstract}

\section{Background}

During development, neurons make networks of connections with other neurons by growing axons and dendrites. These neuronal out-growths are regulated by extracellular cues that signal to cells resulting in phenotypic changes. A major challenge is the identification of molecular mechanisms underlying this highly complex

\footnotetext{
* Correspondence: bchtoohp@nus.edu.sg
'Department of Biochemistry, National University of Singapore, 119260,

* Correspondence: bchtoohp@nus.edu.sg
'Department of Biochemistry, National University of Singapore, 119260, Singapore
}

(c) 2010 Zhou et al; licensee BioMed Central Ltd. This is an Open Access article distributed under the terms of the Creative Commons Attribution License (http://creativecommons.org/licenses/by/2.0), which permits unrestricted use, distribution, and reproduction in any medium, provided the original work is properly cited. and interactive network in terms of the functions of genes and proteins[1].

Currently, transcriptomic methods are widely used as an initial step in unraveling the complex signaling mechanisms underlying physiological and pathological processes and in neuronal differentiation [2-5]. Gene microarray offers a high throughput platform for the analysis of the entire transcriptome to identify differentially expressed genes. Reverse transcription quantitative real-time PCR (RT-qPCR), with a wider dynamic range of quantification and higher assay sensitivity and 
precision, is often used to corroborate microarray findings [6,7]. Regardless of the method used, normalization, a critical process of adjusting the expression measurements between samples to compensate for various sources of variability in the assay, is essential to allow accurate comparisons of the results between different samples and conditions $[8,9]$. Normalization with internal reference gene is used to control for technical and biological variations introduced during both sample preparation and detection by RT-qPCR [10]. It has also been shown to be suitable for the normalization of partially degraded RNA samples [11-13].

With nearly all normalization methods, the assumption that one or more reference genes are constitutively expressed at near-constant levels under all experimental conditions is implicit and the expression levels of all other genes in the sample are then scaled to these reference genes accordingly. It is common to use reference genes selected from an assumed list of "housekeeping" genes (HKGs) which typically include transcripts such as GAPDH and ACTB $[9,10,14]$. A number of studies have now shown that the expressions of these genes, in some but not all experimental conditions, are altered significantly[15-18], thus, making the choice of using these HKGs for normalization uncertain without $a$ priori knowledge.

A variety of approaches have been employed to enable better selection of reference genes. One approach is the use of statistical algorithms, for example, geNorm [14], Best keeper [19], NormFinder [20], Global Pattern Recognition [21], and Equivalence tests [22], to evaluate the relative expression stabilities of genes from a pool of predefined lists of candidates. While this approach is certainly more robust than using the single gene methods, it too is based on potentially unfounded assumptions about which genes may be stably expressed in the conditions studied. These genes are still required to be pre-selected and incorporated into the experimental designs without any a priori evidence to support their use. An alternative and less biased approach is the meta-analysis of large scale gene expression profiles to identify stably expressed genes [23-26]. A selected number of potential references genes can then be validated experimentally and the stability of expressions analyzed by the above mentioned statistical algorithms in defined experimental settings.

To date, reference genes validated for neuronal differentiation studies have not been reported yet. The present study aims to identify suitable reference genes during chemically induced neuronal differentiation of PC12, a cell-line derived from a pheochromocytoma of the rat adrenal medulla. Because of its unique cellular properties, suitability for genetic and biochemical manipulations, the PC12 cell-line is widely regarded as a convenient alternative to endogenous neuronal cells, and serves as a commonly used model system for studies on neuronal differentiation [27-29]. For example, in response to NGF, PC12 cells stop dividing, elaborate neuronal processes, are electrically excitable, and have the potential to form synapses when co-cultured with muscle cells [30]. Here, we measured the temporal expression of twenty novel candidate reference genes identified from microarray studies and the commonly used HKGs, ACTB and GAPDH, at various stages of PC12 differentiation. Based on two independent statistical approaches ["pairwise comparison" [14] and "model based variation" [20], the expressions of ribosomal protein genes RPL19 and RPL29 were found to be highly stable regardless of pharmacological treatments and stages of differentiation. The combination of the two reference genes was sufficient to allow robust and accurate normalization of differentiation related genes.

\section{Results}

\section{Selection of candidate reference genes from microarray} data

It has been suggested that suitable reference genes should be expressed in all experimental conditions and exhibit low coefficient of variation (CV) in their expressions $[25,26,31,32]$. In order to identify such reference genes, we first analyzed the expression profiles of 21,910 genes in naïve PC12 cells and those treated with NGF or GDNF and found 8,568 genes to be expressed in all conditions (detection $\mathrm{p}$ values $<0.05$ ). We then analyzed the top 100 genes with the lowest CV $(0.8 \%-1.45 \%)$ with two well accepted but different statistical approaches, "pairwise comparison" (geNorm) and "model based variation analysis" (NormFinder). The "pairwise comparison" approach assumes that a perfect pair of reference genes has a constant ratio across all experimental conditions. As such, geNorm evaluates the inter-conditional variability of the ratio between each pair of reference genes and calculates a gene stability measure $M$ for each candidate [14]. However, with this method, tightly co-regulated genes will appear to be stable. The second algorithm, NormFinder, was employed to safeguard against such a pitfall of misidentifying expression invariant reference genes. This modelbased variance estimation approach entails analysis of sample subgroups and calculates the variation of each candidate gene individually, based on both intra- and inter-group variation [20]. While geNorm measures relative stability, NormFinder measures absolute stability by decomposing the variance to biological and technical elements. With this method, the expressions of co-regulated genes can be distinguished. Despite the differences in algorithms and assumptions, both statistical methods were in agreement on the identity of the twenty most 
stable genes (Table 1), most of which are novel for the purpose of normalization studies. Interestingly, thirteen of these twenty candidate reference genes were ribosomal protein genes.

\section{Real-time PCR validation of novel candidate reference genes}

As one of the most extensively studied models for neuronal differentiation, PC12 cells respond to a broad spectrum of pharmacological agents, which trigger a myriad of intracellular signaling pathways leading to neuronal differentiation. In order to verify the general utility of the 20 selected putative reference genes (Table 1 ) in a broader range of experimental conditions, we differentiated PC12 cells with other stimuli (Forskolin [33], $\mathrm{KCl}$ [34] and ROCK inhibitor Y27632 [35]) in addition to NGF and GDNF. GDNF was applied to PC12 cells stably expressing GDNF Family Receptor alpha 1a (GFRa1a) and co-receptor RET (either RET9 or RET51 isoforms), which are not endogenously expressed at detectable levels in PC12 cells (data not shown). The percentage of PC12 cells differentiated by the five chemical stimuli was quantified (Figure $1 \mathrm{~A}$ ) and the axonlike features of the extended neurite were confirmed by immunocytochemical analysis with anti-Neurofilament200 antibody (Figure 1B). The extent of neurite outgrowth was highly dependent on the stimuli used. NGF and GDNF stimulation induced longer neurite outgrowths than Forskolin, $\mathrm{KCl}$ or Y27632. Total RNA was collected at $0.5 \mathrm{~h}, 6 \mathrm{~h}, 24 \mathrm{~h}$ and $72 \mathrm{~h}$ from control and treated cells for each stimuli, with biological triplicates, that totaled 120 samples.

We analyzed the expression levels of the aforementioned twenty candidate reference genes, two most commonly used HKGs (GAPDH and ACTB), and three genes which are well known to be regulated by NGF using RT-qPCR (Figure 2). The expression levels of the twenty candidate reference genes and the two HKGs span three orders of magnitude. These reference genes were expressed at comparable levels or lower than the HKGs examined. For accurate determination of interassay variations and primer efficiencies, flanking regions of the genes ( 300 bp) were amplified by PCR, sub-cloned and the sequences verified. These recombinant plasmids were then linearized and served as templates to construct standard curves. All the qPCR assays showed high efficiency of amplification $(>90 \%)$ and low intra- and inter-assay variations (Additional

Table 1 Selection of candidate reference genes from microarray data

\begin{tabular}{|c|c|c|c|c|}
\hline Gene symbol & Definition & Mean & geNorm & NormFinder \\
\hline RPL29 & Ribosomal protein L29 & 14.04 & 1 & 1 \\
\hline RPL10a & Ribosomal protein L10A & 12.49 & 2 & 2 \\
\hline LOC292640 & Vps20-associated 1 homolog & 10.87 & 3 & 3 \\
\hline LOC498143 & Similar to ribosomal protein L15 & 13.71 & 4 & 4 \\
\hline LOC317275 & Similar to ribosomal protein L7-like 1 & 11.88 & 7 & 5 \\
\hline RPS15 & Ribosomal protein S15 & 12.97 & 5 & 6 \\
\hline ARBP & Acidic ribosomal phosphoprotein PO & 14.27 & 6 & 7 \\
\hline RPL14 & Ribosomal protein L14 & 13.89 & 9 & 8 \\
\hline EEF1A1 & Eukaryotic translation elongation factor 1 alpha 1 & 14.17 & 8 & 9 \\
\hline RPS15A & Ribosomal protein S15a & 13.93 & 10 & 10 \\
\hline RPL18 & Ribosomal protein L18 & 13.58 & 11 & 11 \\
\hline REPS1 (P) & RalBP1 associated Eps domain containing protein (predicted) & 10.73 & 12 & 12 \\
\hline LOC363720 & chromatin modifying protein 2B & 10.61 & 14 & 13 \\
\hline CNOT8 & CCR4-NOT transcription complex, subunit 8 & 11.00 & 15 & 14 \\
\hline RTCD1 & RNA terminal phosphate cyclase domain 1 & 10.48 & 17 & 15 \\
\hline RPL19 & Ribosomal protein L19 & 13.74 & 13 & 16 \\
\hline NDUFB6 (P) & NADH dehydrogenase (ubiquinone) 1 beta subcomplex, 6 & 10.43 & 16 & 17 \\
\hline RPL9 & Ribosomal protein L9 & 13.74 & 18 & 18 \\
\hline LOC499803 & Similar to 40 S ribosomal protein $\mathrm{S} 3$ & 13.76 & 19 & 19 \\
\hline RPL3 & Ribosomal protein L3 & 14.03 & 20 & 20 \\
\hline$\overline{\mathrm{ACTB}}$ & Actin, beta & 13.85 & & \\
\hline GAPDH & Glyceraldehyde-3-phosphate dehydrogenase & 12.88 & & \\
\hline
\end{tabular}

Microarray analysis of the expression profiles of 21,910 genes in naïve PC12 cells and those treated with NGF or GDNF for $0.5 \mathrm{~h}$ and $72 \mathrm{~h}$. Twenty candidate reference genes were selected based on pairwise comparison (geNorm) and model based variation (NormFinder) analysis of the top 100 genes with the lowest CV. The log2 transformed values of the average signal intensities among the 24 arrays were shown as Mean. Thirteen of the twenty genes were Ribosomal Protein Genes (Bold). Both ACTB and GAPDH were included for comparison but were not among the top 100 genes recommended by either statistical analysis. 

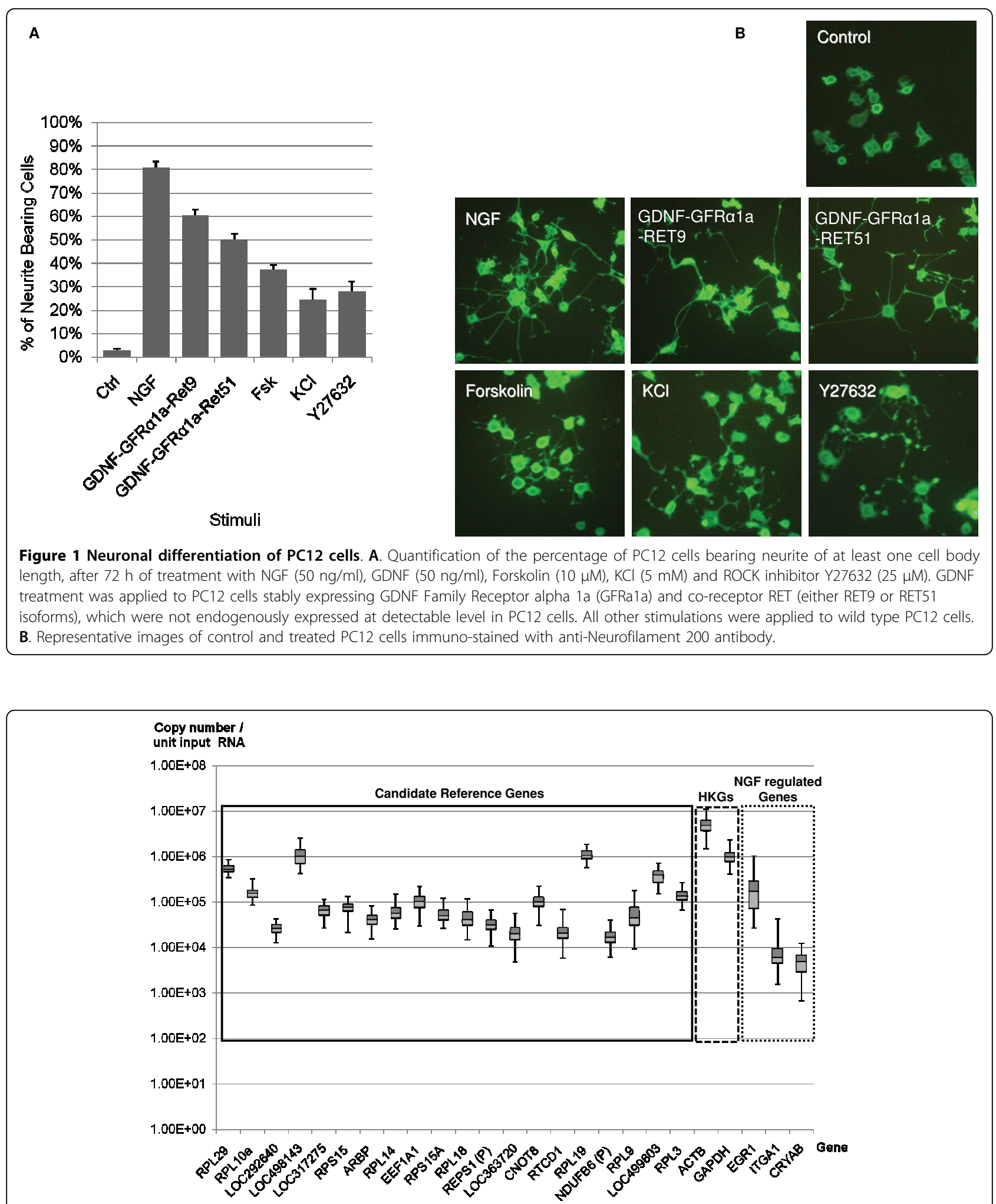

Figure 2 Distribution of the expression levels of genes examined. Box plot representation of the expression levels of twenty candidate reference genes (solid line), two housekeeping genes (dashed line) and three target genes (dotted line) among the 120 biological samples. The expression level of each gene was represented as the absolute copy number per unit input total RNA (0.0625 $\mu \mathrm{g})$, quantified by RT-qPCR using linearized plasmid standards. Primer design, assay efficiency and intra- and inter-assay variations were reported in supplementary data (Additional File 1). 
File 1). All RNA samples showed RQI values of greater than 9, indicative of high quality and integrity (data not shown).

\section{Stabilities of candidate reference genes and common housekeeping genes}

Using both geNorm and NormFinder, we analyzed the expression stabilities of the twenty candidate reference genes and the two commonly used HKGs across all six differentiation conditions. Both statistical approaches recommended the same three ribosomal protein genes RPL19, RPL29 and RPL3 as the overall best reference genes (Figure 3). Pairwise variation analysis by geNorm showed that the combination of RPL19 and RPL29 is sufficiently stable (V2/3 $=0.107$, less than the recommended cut-off of 0.15), thus excluding the need to incorporate a third reference gene RPL3 for normalization of target gene expression. Notably, neither GAPDH nor ACTB were recommended.

Further analysis of candidate gene stabilities in each treatment group (Additional File 2A) or at specific time point (Additional File $2 \mathrm{~B}$ ) revealed that the stability rankings of candidate genes do vary among different subgroups. However, with the exception of RPL29 in $\mathrm{KCl}$ treated samples, the two genes RPL19 and RPL29 were consistently ranked among top 5 in all subgroups. In contrast, the stability rankings of GAPDH and ACTB varied considerably among different subgroups and they were ranked among the least stable ones within the group of 22 genes in several subgroups. The data indicated that the two novel candidate genes RPL19 and RPL29 have higher expression stabilities than both GAPDH and ACTB, and may serve as better normalizers for gene expression in neuronal differentiation of PC12 cells.

\section{Comparison of the normalization factors generated by different reference gene(s)}

To account for possible variations introduced during sample preparation and measurements, raw expression profiles of target genes were scaled by a normalization factor (NF) calculated based on independent measurement of one or more internal reference genes. The variation between NFs generated by different reference

A
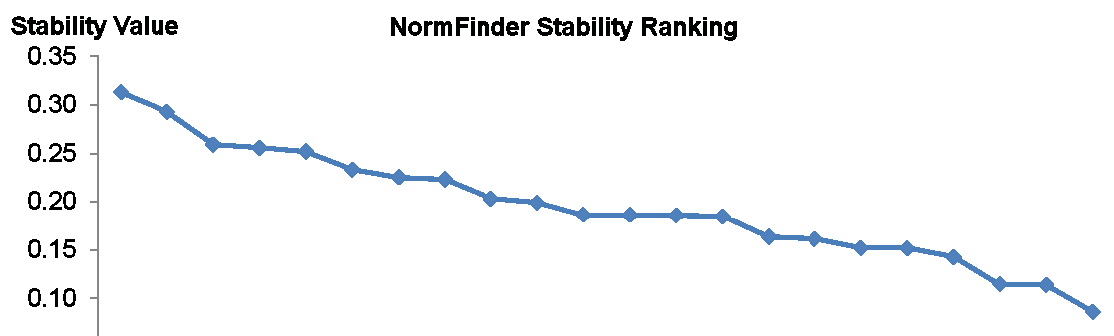

0.05

0.00

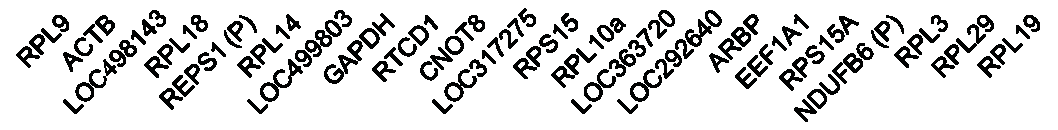

B

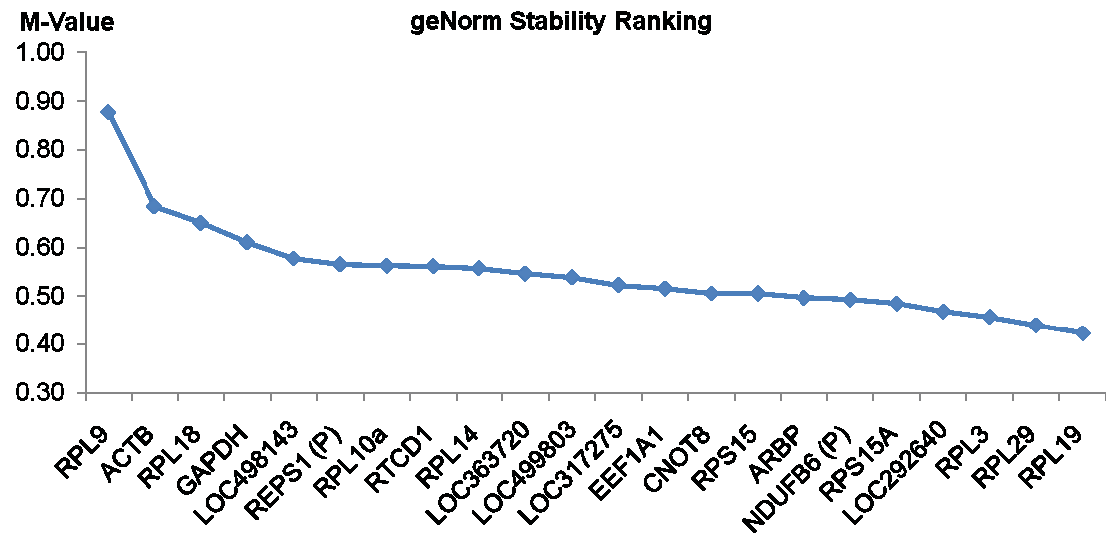

Figure 3 Stability analysis of candidate reference genes and housekeeping genes. Stability rankings of the twenty candidate reference genes and two most commonly used housekeeping genes ACTB and GAPDH, among all 120 biological samples, by NormFinder (A) and geNorm (B). A low 'Stability Value' or 'M-value' correlates to higher gene expression stability. 
genes is thus directly reflective of the variation in the final target gene expression values normalized by different reference genes. We noticed that although RPL19 and RPL29 were ranked as the overall best pair of reference genes, they were not necessarily the best pair for each treatment subgroup. To test the robustness of these two genes across different treatments, we compared the normalization factors calculated based on RPL19 and RPL29 (NF RPL19/RPL29 $)$ to that of the most stable pair of reference genes $\left(\mathrm{NF}_{\text {top } 2}\right)$ in each treatment subgroup. Similarly, we examined the differences between $\mathrm{NF}_{\text {top2 }}$ and NFs calculated based on the commonly used HKGs, ACTB $\left(\mathrm{NF}_{\mathrm{ACTB}}\right)$ or GAPDH $\left(\mathrm{NF}_{\mathrm{GAPDH}}\right)$. The deviations of each $\mathrm{NF}$ from $\mathrm{NF}_{\text {top2 }}$ are represented in Figure 4 (see Additional File 3 for details of calculation). The $\mathrm{NF}_{\mathrm{RPL} 19 / \mathrm{RPL} 29}$ values were found to least deviate from $\mathrm{NF}_{\text {top2 }}$ in $\mathrm{NGF}, \mathrm{GDNF}$ and $\mathrm{KCl}$ subgroups, and had zero deviation in Fsk and Y27632 subgroups as RPL19 and RPL29 were ranked top 2. In contrast, $\mathrm{NF}_{\mathrm{ACTB}}$ and $\mathrm{NF}_{\mathrm{GAPDH}}$ differed substantially from $\mathrm{NF}_{\text {top2 }}$ in many instances, reflective of their varying stabilities across different treatments.

\section{Effect of different reference genes on the interpretation of target gene regulation}

Next, the possibility that using scaling factors of $\mathrm{NF}_{\mathrm{RPL} 19 / 29}, \mathrm{NF}_{\mathrm{ACTB}}$ or $\mathrm{NF}_{\mathrm{GAPDH}}$ may substantially alter the interpretation of target gene expression regulation in NGF induced neuronal differentiation was investigated. The relative fold changes of EGR1, ITGA1 and CRYAB expressions normalized by the three NFs were compared to the values normalized by NF of the top 2 genes
$\left(\mathrm{NF}_{\text {RPL29/RPL10A }}\right)$. No statistically significant differences were observed among $\mathrm{NF}_{\text {RPL29/RPL10A }} \mathrm{NF}_{\text {RPL19/RPL29, }}$ and $\mathrm{NF}_{\mathrm{ACTB}}$ normalized values; whereas $\mathrm{NF}_{\mathrm{GAPDH}}$ normalized fold changes were significantly different (Figure $5 \mathrm{~A}-\mathrm{C})$. In the case of EGR1 and ITGA1, the use of GAPDH as reference gene resulted in the underestimation of target genes expressions, leading to false negative conclusions when a two-fold cut off was applied (Figure $5 \mathrm{~A}-\mathrm{B})$. On the other hand, normalization by GAPDH resulted in the significant over-estimation of the downregulation of CRYAB in NGF treated samples (Figure $5 \mathrm{C})$.

The clearly different expression profiles of EGR1, ITGA1, and CRYAB when normalized to GAPDH raised the possibility that GAPDH expression could be regulated over the course of NGF induced differentiation. Normalization of GAPDH expression by the NF of the top 2 genes $\left(\mathrm{NF}_{\mathrm{RPL} 29 / \mathrm{RPL} 10 \mathrm{~A}}\right)$ and the $\mathrm{NF}_{\mathrm{RPL} 19 / \mathrm{RPL} 29}$, revealed that GAPDH expression was indeed significantly elevated ( $>2.5$ fold at $24 \mathrm{~h}$ ) in NGF-stimulated PC12 cells (Figure 6). A more detailed analysis of the kinetics of GAPDH expression over time revealed that expression of GAPDH indeed increased over a period of $28 \mathrm{~h}$ (Additional File 4). As a result, the use of GAPDH as a single, unverified reference gene would invariably lead to erroneous interpretation of target gene regulation.

Similarly, we investigated the effect of different reference gene(s) on normalized target gene expressions in GDNF, Forskolin, $\mathrm{KCl}$ and ROCK inhibitor Y27632 treated samples. Similar to the case of NGF treatment, with GDNF stimulated PC12-GFRa1a/RET9 and PC12-

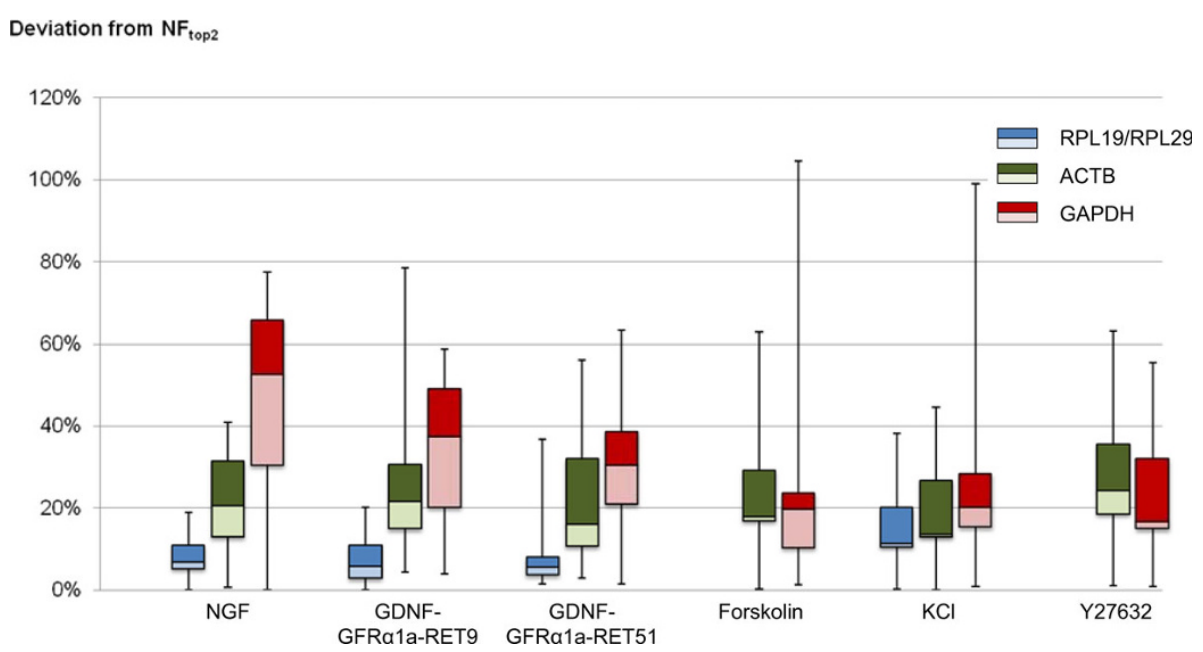

Figure 4 Comparison of the normalization factors calculated using different reference gene(s). Normalization factors (NFs) calculated with RPL19/RPL29, ACTB and GAPDH were compared to that calculated by the top 2 reference genes $\left(N F_{\text {top } 2}\right)$ as recommended by both NormFinder and geNorm, for each stimulus. The percentage deviations of $\mathrm{NF}_{\mathrm{RPL19} / \mathrm{RPL} 29 ;} \mathrm{NF}_{\mathrm{ACTB}} ; \mathrm{NF}_{\text {GAPDH }}$ from $\mathrm{NF}_{\text {top2 }}\left(\mid \mathrm{NF}_{\mathrm{x}}-\mathrm{NF}_{\text {top2 } 2} / \mathrm{NF}_{\text {top2 }}\right)$ were represented by box plot. The $25^{\text {th }}$ percentile to the $75^{\text {th }}$ percentile (boxes), and ranges (whiskers) were shown. 


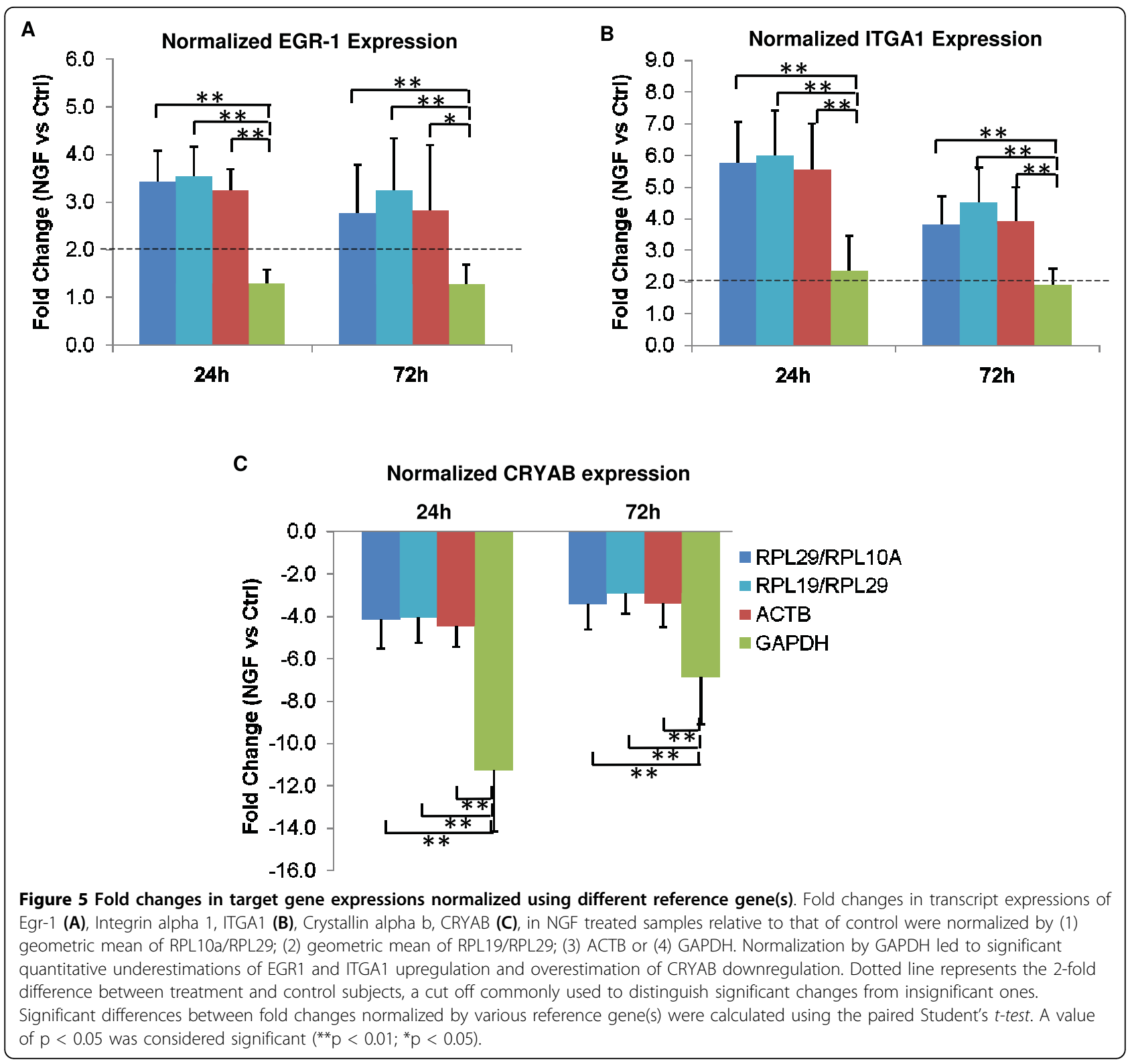

GFRa1a/RET51 cells, normalization by GAPDH resulted in the underestimations of GDNF induced upregulation of EGR1 and ITGA1 expressions; and over-estimated CRYAB down-regulation (Additional File 5A, B). Interestingly, normalization by ACTB was found to overestimate the expression of EGR1 and ITGA1 expressions in PC12-GFRa1a/RET51 but not RET9 cells (Additional File 5B, $72 \mathrm{~h}$ ), highlighting the subtle differences between GFRa1a/RET9 and GFRa1a/RET51 systems.

For Forskolin and ROCK inhibitor Y23672 differentiated samples, normalization by ACTB consistently led to the over-estimations of target gene expressions (Additional File 5C, D). Depending on the time point analyzed, normalization by GAPDH was shown to result in either underestimations or overestimations of target gene expressions (Additional File 5C, D). In $\mathrm{KCl}$ treated samples, no statistical significant difference was observed among $\mathrm{NF}_{\text {top2 }}$ (RPL19/REPS1), NF $\mathrm{RPL19/RPL29}_{\text {, }}$ $\mathrm{NF}_{\mathrm{ACTB}}$ and $\mathrm{NF}_{\mathrm{GAPDH}}$ normalized target gene expression, which suggested that all four were acceptable reference gene(s) for this particular experimental condition (data not shown).

It is thus evident that the stabilities of the two most commonly used HKGs, GAPDH and ACTB vary across different experimental conditions during neuronal differentiation of PC12 cells. They were acceptable reference genes under some conditions but may significantly under- or over-estimate target gene expression under 


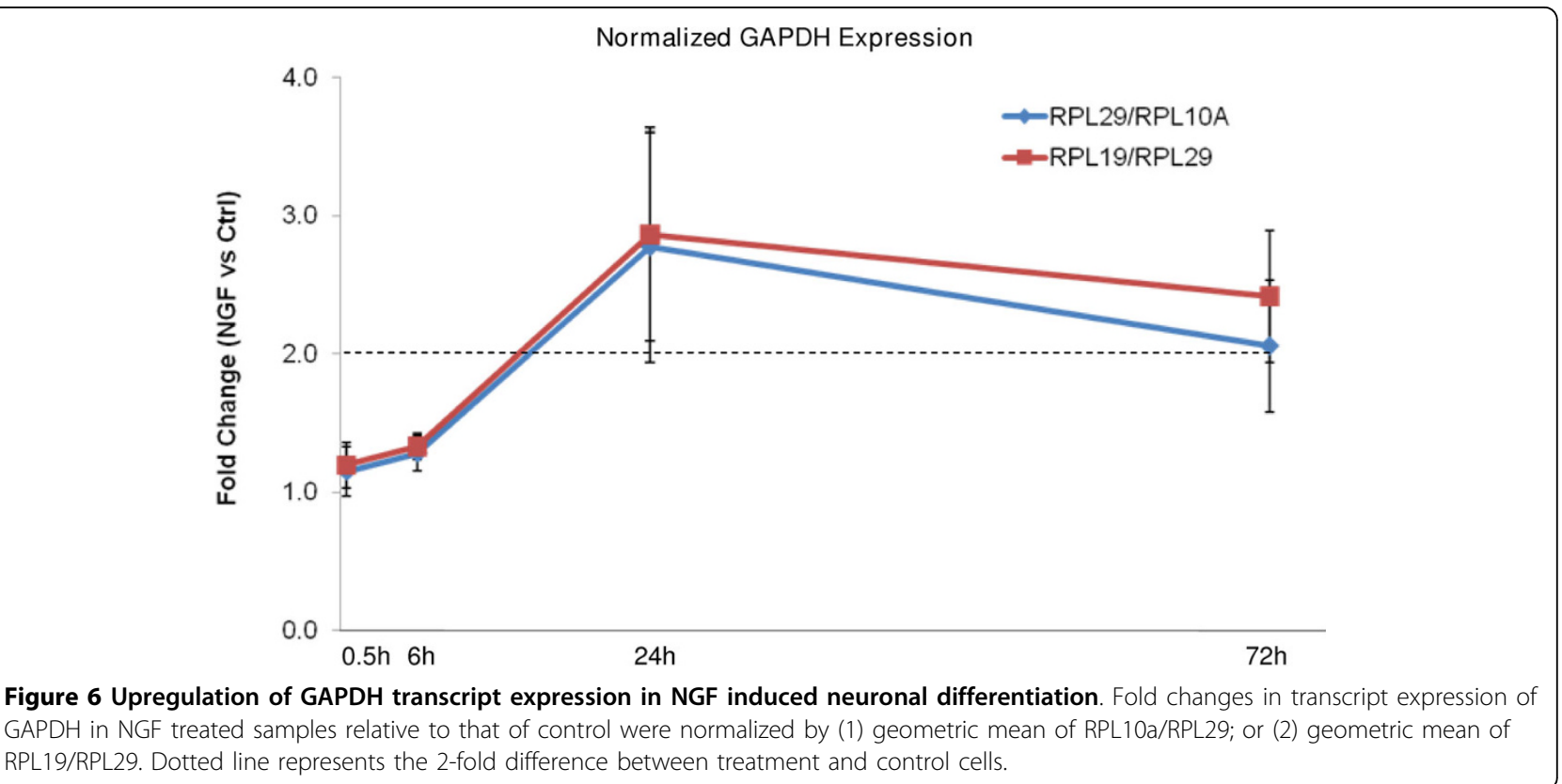

others. On the contrary, the two novel candidate reference genes RPL19 and RPL29 were stably expressed among all conditions analyzed and allowed accurate normalization of differentially regulated genes during PC12 differentiation. It is worthy to note that at early time points $(0.5 \mathrm{~h}$ and $6 \mathrm{~h})$, the expressions of EGR1, ITGA1 and CRYAB did not show any significant differences when scaled with either $\mathrm{NF}_{\text {top2, }} \mathrm{NF}_{\mathrm{RPL} 19 / \mathrm{RPL} 29}$, $\mathrm{NF}_{\mathrm{ACTB}}$ or $\mathrm{NF}_{\mathrm{GAPDH}}$ (data not shown). This is consistent with the observation that the expression of GAPDH did not change significantly at the early time points (Figure 6. and Additional File 4).

\section{Discussion}

Twenty candidate reference genes that showed little variation but high expression in PC12 cells differentiated with NGF and GDNF were first selected from microarray datasets using two independent statistical algorithms. Together with two well studied HKGs, the expression stabilities of these candidate reference genes were further analyzed using RT-qPCR in cells differentiated with other stimuli. From these studies, unexpectedly, RPL19 and RPL29 but not the HKGs, were identified as suitable reference genes that can be used for normalization of gene expression in neuronal differentiation of PC12 induced by a variety of chemical stimuli.

Neuronal differentiation is a process where cells undergo enormous morphological changes, over a period of several days. It is accompanied by substantial biochemical changes including cell cycle exit [36], changes in metabolism $[37,38]$ and alteration in structural proteins $[39,40]$. Since the commonly used reference genes are mostly structural proteins or enzymes involved in metabolism, it is especially important to validate the stabilities of these genes during the process of differentiation. Many of these studies investigated gene expression changes in PC12 but few have evaluated the suitability of HKGs as normalizers in this model. Our microarray analysis revealed that a group of novel candidate genes was more stably expressed than commonly used HKGs ACTB and GAPDH, suggesting that ACTB and GAPDH may not be ideal reference genes in neuronal differentiation of PC12 cells.

In an effort to gain an insight into the temporal regulation of genes during neuronal differentiation, it is necessary that the reference genes used are stably expressed over a period of days. GAPDH and ACTB have been used for normalization in more than $90 \%$ of previous reports [41], often without proper validation of their stabilities. Numerous publications have reported that such HKGs can be differentially expressed under various experimental paradigms and are therefore inappropriate for normalization $[9,15,16]$. However, there are also recent reports that these HKGs are stably expressed and can serve as reference genes $[17,18,42]$. Most genes, including GAPDH and $\mathrm{ACTB}$, examined in this study were stable at early stages of differentiation $(0.5 \mathrm{~h}$ or $6 \mathrm{~h})$. However, as differentiation proceeded with dramatic morphological changes and concomitant biochemical changes, the instability of expressions of GAPDH and many of the genes examined was obvious. In the case of GAPDH, this instability issue correlated well with the temporal increase of expression level, which peaked at $28 \mathrm{~h}$ and was sustained over a period of $72 \mathrm{~h}$. While GAPDH may still serve as a reference gene for PC12 cells under specific conditions, the validity of 
using this gene and other less stable ones should be experimentally verified. However, the two RP genes (RPL19 and RPL29) that showed good stability in expression over the period of differentiation provided an optimal pair of reference genes for the entire period of and various experimental conditions for neuronal differentiation.

Among the twenty candidate genes selected, thirteen were ribosomal protein genes, suggesting that the family of ribosomal protein genes may become yet another source of reference genes. Several recent publications have validated and recommended the use of ribosomal protein genes as reference genes [23,25], while others have reported their tissue-dependent variations [43]. A plausible explanation for such disparity is the large number of ribosomal protein genes present in mammalian systems (80 genes in human, mouse and rat genome), which may be stably or differentially expressed depending on the tissue type or experimental conditions. At present, relatively little is known about these mammalian ribosomal proteins, as compared to their bacterial and archael counterparts [44]. While bacterial ribosomal protein genes exist largely in clusters, the mammalian RP genes are dispersed throughout the genome [45]. Some have suggested that all of these proteins are intimately involved in ribosome production and could be co-regulated. Depletion of a particular ribosomal protein would generally cause a reduction of all other ribosomal proteins in the same ribosome sub-unit [46]. Other reports have shown that some ribosomal protein genes could be regulated independent of others [47]. Recently, extra-ribosomal functions of some of these proteins have been reported [48-51], suggesting that they may be individually regulated. A previous study comparing random ESTs from naïve and NGFtreated PC12 cells, reported an NGF-promoted decrease in the expressions of RPL19 [52]. However, this decrease in RPL19 was not observed in other studies using SAGE [53] or microarray [2]. Similar to the latter studies, we too did not observe changes in RPL19 transcripts with NGF-treated PC12. Moreover, the SAGE study but not the microarray analysis reported a significant decrease in RPL29 expression. Using both microarray and RTqPCR, we have also shown that RPL29 was unchanged when the cells were differentiated. The reasons for these discrepancies are unclear and may be due to the differences in methods used. We have shown here by quantitative real-time PCR that some ribosomal protein genes, RPL19 and RPL29, are highly stably expressed and are thus suitable reference genes, whereas others like RPL9 and RPL18 can vary significantly during differentiation.

Unlike some studies that attempted to identify ideal reference genes through meta-analysis of many publically available microarray data which includes a diverse range of tissue types and experimental conditions, this study was designed to specifically identify a set of suitable reference genes for PC12 cells undergoing neuronal differentiation. We have performed both the microarray analysis and RTqPCR validation on biological samples prepared with the same techniques and reagents, thus minimizing variations introduced by differences in sample preparation methods and assay platforms. We have also systematically evaluated the effect of the use of NFs of inappropriate reference gene(s) on the expression changes of the target genes and the erroneous results they resulted in. Thus, with neuronal differentiation of $\mathrm{PC} 12$ cells, scaling with the geometric means of the expressions of RPL19 and RPL29 is recommended for the accurate normalization of gene expression. Whether these two genes are suitable for normalization of neuronal differentiation in other systems remains to be evaluated.

\section{Conclusions}

Twenty novel candidate reference genes were identified and their expression stabilities were analyzed and compared to that of commonly used HKGs ACTB and GAPDH. Through this systematic study that included both microarray analysis and RT-qPCR, we have found two ribosomal protein genes RPL19, and RPL29 to be stably expressed during neuronal differentiation of PC12 cells, induced by five different chemical stimuli, over 72 $h$. The combination of these two novel reference genes allowed robust and accurate normalization of differentially expressed genes, regardless of stimuli and stages of differentiation. In contrast, the use of an inappropriate reference gene like GAPDH led to significant erroneous estimation of differentially expressed genes.

\section{Methods}

\section{Cell Culture}

The rat pheochromocytoma cell line PC12 (catalog \# CRL-1721; American Type Culture Collection) cells were grown in DMEM supplemented with $10 \%$ heatinactivated fetal bovine serum (FBS; Hyclone, Logan, UT) and 5\% Horse Serum (HS), in a humidified atmosphere with $5 \% \mathrm{CO}_{2}$ at $37^{\circ} \mathrm{C}$. Wild type $\mathrm{PC} 12$ cells, that do not endogenously express GFRa or RET (data not shown), were co-infected with murine GFRa1a (NM_010279) and RET9 (NM_001080780) or RET51 (NM_009050) in pQCXIN or pQCXIH vector by retroviral infection (Clontech, Mountain View, CA) and selected with $0.4 \mathrm{mg} / \mathrm{ml} \mathrm{G} 418$ and $0.1 \mathrm{mg} / \mathrm{ml}$ Hygromycin, over a period of 2 months.

\section{Differentiation, sample collection and assessment of neurite outgrowth}

Two million wild type or infected PC12 cells were seeded in $75 \mathrm{~cm}^{2}$ flask (NUNC, Finland) overnight, in DMEM supplemented with $10 \% \mathrm{FBS}$ and $5 \% \mathrm{HS}$, 
followed by serum depletion for $12 \mathrm{~h}$. PC12 cells were then treated with $50 \mathrm{ng} / \mathrm{ml}$ recombinant human GDNF (Peprotech, NJ), 50 ng NGF (Peprotech, NJ), $10 \mu \mathrm{M}$ Forskolin (Sigma, St. Louis, MO), $5 \mathrm{mM} \mathrm{KCl} \mathrm{(Sigma,} \mathrm{St.}$ Louis, MO) or $25 \mu \mathrm{M}$ ROCK inhibitor Y27632 (Calbiochem, USA) in DMEM to induce neurite outgrowth. Total RNA was isolated from control and treated cells at $0.5,6,24$ or $72 \mathrm{~h}$. For neurite outgrowth assessment, cells bearing at least one neurite with the length equivalent to the cell bodies were scored at $72 \mathrm{~h}$ by independent observers. More than 400 cells from three different fields were counted per flask.

\section{Immunocytochemistry}

Control and treated PC12 Cells were fixed with 4\% paraformaldehyde in $1 \mathrm{xPBS}$ for $15 \mathrm{~min}$ at $37^{\circ} \mathrm{C}$, and subsequently in methanol at $-20^{\circ} \mathrm{C}$ for an additional $15 \mathrm{~min}$. After three washes with $1 x$ PBS, cells were permeabilized and blocked with normal goat serum (1:10; Dako, Glostrup, Denmark) in $0.5 \%$ Triton X-100/PBS for 30 $\min$ at $37^{\circ} \mathrm{C}$. The cells were then incubated with highmolecular-weight neurofilament protein (NF-200) antibody (Sigma, St. Louis, MO) at 1:80 dilution in $0.1 \%$ Triton $\mathrm{X}-100 / 0.1 \% \mathrm{BSA} / 1 \mathrm{xPBS}$ for $1 \mathrm{~h}$ at $37^{\circ} \mathrm{C}$ and washed three times in $1 x P B S$. Subsequently, the cells were incubated with goat anti-rabbit fluorescent secondary antibody (Alexa Fluor 488; Invitrogen, CA) diluted $1: 1000$ in $0.1 \%$ Triton X-100/0.1\% BSA/1xPBS for $1 \mathrm{~h}$. The cells were then washed three times in $1 x P B S$. Image acquisition was performed using the Zeiss inverted Axovert 25 microscope equipped with fluorescence detection (Oberkochen, Germany).

\section{RNA Purification and cDNA Preparation}

Total RNA from PC12 cells was prepared using TRIzol reagent (Invitrogen, CA) according to manufacturer's instruction. Total RNA was collected from samples in quadruplicate at each treatment time point and the integrity of the RNA validated by denaturing agarose gel electrophoresis and using the StdSens analysis chip on the Experion Automated Electrophoresis System (BioRad, CA) according to manufacturer's instructions. The Experion Automated Electrophoresis System assigns a RQI to each RNA electropherogram which ranges from 10 (intact RNA) to 1 (completely degraded RNA). RNA concentration was quantified using a NanoDrop ND-1000 spectrophotometer (Thermo Scientific, Wilmington, DE), and the $260 / 280$ and $260 / 230$ ratios were examined for protein and solvent contamination. Five micrograms of total RNA were reverse transcribed in a total volume of $20 \mu \mathrm{l}$ containing $400 \mathrm{U}$ of ImpromII and $0.5 \mu \mathrm{g}$ of random hexamer (Promega, Madison, WI) for $60 \mathrm{~min}$ at $42^{\circ} \mathrm{C}$ according to the manufacturer's instructions. The reaction was terminated by heating at $70^{\circ} \mathrm{C}$ for $5 \mathrm{~min}$, and the cDNA was diluted 1:20 for quantitative real-time PCR.

\section{Microarray}

PC12 cells were seeded on $25 \mathrm{~cm}^{2}$ flask in complete medium and subsequently incubated for $12 \mathrm{~h}$ in serum free DMEM. The cells were then treated with GDNF (50 ng/ $\mathrm{ml}$ ) or NGF $(50 \mathrm{ng} / \mathrm{ml})$ for $0.5 \mathrm{~h}$ or $72 \mathrm{~h}$ in duplicates. Total RNA was isolated, quantified and integrity verified before it was amplified using Ambion Illumina RNA Amplification kit (Ambion, TX, USA). Briefly, total RNA (500 ng) was reverse transcribed by ArrayScript in the presence of T7 Oligo(dT) primer. Second strand of the cDNA was synthesized by DNA polymerase at $16^{\circ} \mathrm{C}$ for $2 \mathrm{~h}$. The cDNA was purified and in vitro transcribed with T7 RNA polymerase and biotin-NTPs. Biotin-labeled cRNA samples were purified and quantified by ND-1000 spectrophotometer (NanoDrop, Fisher Thermo, DE, USA). Each cRNA (750 ng) was hybridized to RatRef-12 Expression BeadChip (Illumina, San Diego, CA, USA) containing 22,523 probes for a total of 21,910 rat genes selected primarily from the NCBI RefSeq database (Release 16) according to instruction provided by Experienced User Card (11286340 Rev A, Illumina). After hybridization, washing and blocking, the BeadChip was incubated with Streptavidin-Cy3 solution (Amersham Biosciences, Piscataway, NJ, USA). Fluorescent signals were obtained from scans on the high resolution Illumina BeadArray reader, using a two-channel, $0.8 \mu \mathrm{m}$ resolution confocal laser scanner. The Illumina BeadStudio software (Version 2.0) was used to extract fluorescence intensities and the raw fluorescent data was background subtracted and used for analysis. Background is defined as the average signal intensity estimated from the negative control bead types. Outliers are removed using the median absolute deviation method. Detection p-values produced by the BeadStudio software were corrected for multiple hypothesis testing.

\section{Primer Design and Plasmid Standards}

The Genbank accession for each candidate reference gene was retrieved from the Illumina microarray probe set and compared to the NCBI RefSeq database (Release 16; http://www.ncbi.nlm.nih.gov. Transcript splicing sites were retrieved from Ensembl http://www.ensembl. org. Where more than one transcript matched the probe, the sequences were aligned and the primers were designed to amplify the consensus region. Vector NTI Advance 10 was used to design two sets of primers for each target gene. The first set of primers generates an amplicon of $\sim 300 \mathrm{bp}$ and is used as a template for RTqPCR of the targeted gene. The template was subcloned into pGEMT-easy (Promega) vector as previously described [54]. The second set of primers was used for RT-qPCR and was designed to amplify a $~ 100$ bp region 
within the $\sim 300 \mathrm{bp}$ template. Both primer sets were exon spanning to avoid amplification from genomic sequences. Where possible, primers for RT-qPCR were designed to target the same exons used in the Illumina Expression BeadChip. All primer sequences were evaluated for possible false priming to known rat sequences using the NCBI BLAST tool http://blast.ncbi.nlm.nih. gov/. All products generated after amplifications were verified by gel-electrophoresis and DNA sequencing.

\section{Quantitative Real-Time PCR}

Real-time PCR was performed on Biorad iCycler 4 Real-Time PCR Detection System (Bio-Rad, Hercules, CA) using SYBR Green I. The threshold cycles (Ct) were calculated using the iQ5 Optical system software version 2.0. Real-time PCR was performed after an initial denaturation for $10 \mathrm{~min}$ at $95^{\circ} \mathrm{C}$, followed by 40 cycles of $30 \mathrm{~s}$ denaturation at $95^{\circ} \mathrm{C}, 30 \mathrm{~s}$ annealing at $60^{\circ} \mathrm{C}$, and $30 \mathrm{~s}$ extension at $72^{\circ} \mathrm{C}$. Fluorescent detection was performed at the annealing phase. Melt curve analysis was carried out at the end of the cycling to confirm that a single product had been amplified. Primer dimer formation in all the assays showed distinct melt characteristics from the correct amplicons. The reaction was performed in a total volume of $40 \mu \mathrm{l}$ in $1 \times$ XtensaMix-SG (BioWORKS, Singapore), containing 2.5 $\mathrm{mM} \mathrm{MgCl}{ }_{2}, 10$ pmol of each primer, and $0.5 \mathrm{U}$ of KlearTaq Hotstart DNA polymerase (KBioscience, UK). All real-time PCR quantification was performed simultaneously with linearized plasmid standards and a nontemplate control [54]. As PCR is an exponential process, it can be described by the equation, $\mathrm{N}_{\mathrm{n}}=\mathrm{N}_{0}(1+$ $\varepsilon)^{\mathrm{n}}$, where $\mathrm{N}_{\mathrm{n}}$ is the number of target molecules at cycle $\mathrm{n}, \mathrm{N}_{0}$ is the initial number of target molecules, $\varepsilon$ is the efficiency of amplification and $n$ is the number of cycles. The efficiency of target amplification of an assay was determined from the slope of a plot of $\mathrm{Ct}$ (Threshold cycle) versus $-\log _{10}$ concentration of the initial number of target molecules. High efficiency of amplification has a slope approaching the value of 3.32 cycles for every 10 -fold dilution of the target. The gene expression levels were interpolated from standard curves and expressed as absolute copy numbers. All Real-time PCR experiments were compliant with the MIQE (Minimum Information for Publication of Quantitative Real-Time PCR Experiments) guidelines [55].

\section{Statistical data analysis}

Gene expression stability analysis using two publicly available software tools, geNorm http://medgen.ugent. be/genorm/ and NormFinder http://www.mdl.dk/, were carried out according to authors' instruction.
Additional file 1: RT-qPCR assay design and performance. Efficiencies of amplification and inter/intra-assay precisions of the assays used to measure the twenty candidate reference genes, two commonly used housekeeping genes and three target genes quantified in this study. Click here for file

[http://www.biomedcentral.com/content/supplementary/1471-2164-1175-S1.ZIP]

Additional file 2: Stability rankings of twenty candidate reference genes, ACTB and GAPDH in treatment and time-point subgroups. Stability rankings were determined by NormFinder (Italic) and geNorm, for each stimulus (Additional File 2A) or time point (Additional File 2B)

subgroup. The top two candidate genes (RPL19 and RPL29) in overall ranking (Figure 4) were bolded in red and the two HKGs were bolded and highlighted in grey.

Click here for file

[http://www.biomedcentral.com/content/supplementary/1471-2164-1175-S2.ZIP ]

Additional file 3: Calculations of the deviation from $\mathrm{NF}_{\text {top2 }}$.

Illustration of the calculation of the deviation of different normalization factors $\left(\mathrm{NF}_{\mathrm{RPL} \text { 19/RPL29; }} \mathrm{NF}_{\text {ACTB }}\right.$ and $\left.\mathrm{NF}_{\text {GAPDH }}\right)$ from $\mathrm{NF}_{\text {top2 }}\left(\mathrm{NF}_{\text {RPL10ARPL29 }}\right.$ for NGF group), in 12 control and 12 NGF treated samples.

Click here for file

[http://www.biomedcentral.com/content/supplementary/1471-2164-1175-S3.PDF ]

Additional file 4: Time course analysis of GAPDH expression in NGF induced PC12 differentiation. A detailed time course analysis showing the up-regulation of GAPDH transcript expression by NGF treatment in PC12 cells, normalized by the geometric mean of RPL19 and RPL29. Click here for file

[http://www.biomedcentral.com/content/supplementary/1471-2164-1175-S4.PDF ]

Additional file 5: Normalized target gene expression regulation in PC12 cells differentiated with GDNF, Forskolin and Y27632. Fold changes in transcript expressions of Egr-1 (i), Integrin alpha 1, ITGA1 (ii), and Crystallin alpha b, CRYAB (iii), in GDNF-GFRala-RET9 (A), GDNFGFRa1a-RET51 (B), Forskolin (C), Y27632 (D) treated samples relative to that of control were normalized by geometric mean of top 2 reference genes in each subgroup; geometric mean of RPL19/RPL29; ACTB or GAPDH. Normalization by ACTB resulted in the over-estimation of target gene expression. Normalization by GAPDH led to either under- or overestimation of target gene expression. Dotted line represents the 2-fold difference between treatment and control subjects, a cut off commonly used to distinguish significant changes from insignificant ones.

Significant differences between fold changes normalized by various reference gene(s) were calculated using the paired Student's $t$ test. A value of $p<0.05$ was considered significant $\left({ }^{* *} p<0.01 ;{ }^{*} p<0.05\right)$ Click here for file

[http://www.biomedcentral.com/content/supplementary/1471-2164-1175-S5.DOCX]

\section{Acknowledgements}

The authors would like to thank NUS Department of Biochemistry for providing the necessary laboratory equipments and administrative support. The authors would like to acknowledge Ms Chen Peiyi from NUS Department of Statistics and Applied Probability for her help with the statistical analysis.

\section{Author details}

'Department of Biochemistry, National University of Singapore, 119260, Singapore. ${ }^{2}$ Chemical Pharmaceutical Engineering, Singapore-Massachusetts Institute of Technology Alliance, 117576, Singapore.

\section{Authors' contributions}

LZ carried out most of the sample preparation and real-time PCR, performed statistical analysis of the data, participated in the microarray analysis and drafted the manuscript. QEL designed the primers, performed the molecular cloning, and participated in sample preparation, real-time PCR and data 
analysis. GW performed the microarray analysis, participated in sample preparation and real-time PCR. HPT conceived of the study, and participated in its design, coordination, pipetting of samples and reviewed the manuscript. All authors read and approved the final manuscript.

Received: 24 August 2009

Accepted: 29 January 2010 Published: 29 January 2010

\section{References}

1. Qiu Z, Ghosh A: A brief history of neuronal gene expression: regulatory mechanisms and cellular consequences. Neuron 2008, 60(3):449-455.

2. Dijkmans TF, van Hooijdonk LW, Schouten TG, Kamphorst JT, Vellinga AC, Meerman JH, Fitzsimons CP, De Kloet ER, Vreugdenhil E: Temporal and functional dynamics of the transcriptome during nerve growth factorinduced differentiation. Journal of neurochemistry 2008, 105(6):2388-2403.

3. Perez-Iratxeta C, Palidwor G, Porter CJ, Sanche NA, Huska MR, Suomela BP, Muro EM, Krzyzanowski PM, Hughes E, Campbell PA, et al: Study of stem cell function using microarray experiments. FEBS letters 2005, 579(8):1795-1801.

4. Rhodes DR, Chinnaiyan AM: Integrative analysis of the cancer transcriptome. Nature genetics 2005, 37(Suppl):S31-37.

5. Preuss TM, Caceres M, Oldham MC, Geschwind DH: Human brain evolution: insights from microarrays. Nat Rev Genet 2004, 5(11):850-860.

6. Rajeevan MS, Ranamukhaarachchi DG, Vernon SD, Unger ER: Use of realtime quantitative $P C R$ to validate the results of CDNA array and differential display PCR technologies. Methods (San Diego, Calif) 2001, 25(4):443-451.

7. Chuaqui RF, Bonner RF, Best CJ, Gillespie JW, Flaig MJ, Hewitt SM, Phillips JL, Krizman DB, Tangrea MA, Ahram M, et al: Post-analysis followup and validation of microarray experiments. Nature genetics 2002, 32(Suppl):509-514.

8. Do $\mathrm{JH}$, Choi DK: Normalization of microarray data: single-labeled and dual-labeled arrays. Molecules and cells 2006, 22(3):254-261.

9. Huggett J, Dheda K, Bustin S, Zumla A: Real-time RT-PCR normalisation; strategies and considerations. Genes and immunity 2005, 6(4):279-284.

10. Thellin O, Zorzi W, Lakaye B, De Borman B, Coumans B, Hennen G, Grisar T, Igout $A$, Heinen $E$ : Housekeeping genes as internal standards: use and limits. Journal of biotechnology 1999, 75(2-3):291-295.

11. Antonov J, Goldstein DR, Oberli A, Baltzer A, Pirotta M, Fleischmann A, Altermatt $H J$, Jaggi R: Reliable gene expression measurements from degraded RNA by quantitative real-time PCR depend on short amplicons and a proper normalization. Laboratory investigation; a journal of technical methods and pathology 2005, 85(8):1040-1050.

12. Fleige $S$, Pfaffl MW: RNA integrity and the effect on the real-time qRTPCR performance. Molecular aspects of medicine 2006, 27(2-3):126-139.

13. Fleige $S$, Walf $V$, Huch S, Prgomet C, Sehm J, Pfaffl MW: Comparison of relative mRNA quantification models and the impact of RNA integrity in quantitative real-time RT-PCR. Biotechnology letters 2006, 28(19):1601-1613.

14. Vandesompele J, De Preter K, Pattyn F, Poppe B, Van Roy N, De Paepe A, Speleman F: Accurate normalization of real-time quantitative RT-PCR data by geometric averaging of multiple internal control genes. Genome biology 2002, 3(7):RESEARCH0034.

15. Waxman $\mathrm{S}$, Wurmbach E: De-regulation of common housekeeping genes in hepatocellular carcinoma. BMC genomics 2007, 8:243.

16. Jung M, Ramankulov A, Roigas J, Johannsen M, Ringsdorf M, Kristiansen G, Jung $\mathrm{K}:$ In search of suitable reference genes for gene expression studies of human renal cell carcinoma by real-time PCR. BMC molecular biology 2007, 8:47.

17. Rhinn H, Marchand-Leroux C, Croci N, Plotkine M, Scherman D, Escriou V: Housekeeping while brain's storming Validation of normalizing factors for gene expression studies in a murine model of traumatic brain injury. BMC molecular biology 2008, 9:62.

18. Spinsanti G, Panti C, Lazzeri E, Marsili L, Casini S, Frati F, Fossi CM: Selection of reference genes for quantitative RT-PCR studies in striped dolphin (Stenella coeruleoalba) skin biopsies. BMC molecular biology 2006, 7:32.

19. Pfaffl MW, Tichopad A, Prgomet C, Neuvians TP: Determination of stable housekeeping genes, differentially regulated target genes and sample integrity: BestKeeper-Excel-based tool using pair-wise correlations. Biotechnology letters 2004, 26(6):509-515.

20. Andersen $C L$, Jensen $J \mathrm{~L}$, Orntoft TF: Normalization of real-time quantitative reverse transcription-PCR data: a model-based variance estimation approach to identify genes suited for normalization, applied to bladder and colon cancer data sets. Cancer research 2004, 64(15):5245-5250.

21. Akilesh S, Shaffer DJ, Roopenian D: Customized molecular phenotyping by quantitative gene expression and pattern recognition analysis. Genome research 2003, 13(7):1719-1727.

22. Haller F, Kulle B, Schwager S, Gunawan B, von Heydebreck A, Sultmann H, Fuzesi $L$ : Equivalence test in quantitative reverse transcription polymerase chain reaction: confirmation of reference genes suitable for normalization. Analytical biochemistry 2004, 335(1):1-9.

23. Popovici V, Goldstein DR, Antonov J, Jaggi R, Delorenzi M, Wirapati P. Selecting control genes for RT-QPCR using public microarray data. BMC bioinformatics 2009, 10:42.

24. Lee S, Jo M, Lee J, Koh SS, Kim S: Identification of novel universal housekeeping genes by statistical analysis of microarray data. Journal of biochemistry and molecular biology 2007, 40(2):226-231.

25. de Jonge HJ, Fehrmann RS, De Bont ES, Hofstra RM, Gerbens F, Kamps WA, De Vries EG, Zee van der AG, te Meerman GJ, ter Elst A: Evidence based selection of housekeeping genes. PLOS ONE 2007, 2(9):e898.

26. Kwon MJ, Oh E, Lee S, Roh MR, Kim SE, Lee Y, Choi YL, In YH, Park T, Koh SS, et al: Identification of novel reference genes using multiplatform expression data and their validation for quantitative gene expression analysis. PLoS ONE 2009, 4(7):e6162.

27. Ravni A, Bourgault S, Lebon A, Chan P, Galas L, Fournier A, Vaudry H, Gonzalez B, Eiden LE, Vaudry D: The neurotrophic effects of PACAP in PC12 cells: control by multiple transduction pathways. Journal of neurochemistry 2006, 98(2):321-329.

28. Levi A, Biocca S, Cattaneo A, Calissano P: The mode of action of nerve growth factor in PC12 cells. Molecular neurobiology 1988, 2(3):201-226.

29. Vaudry D, Stork PJ, Lazarovici P, Eiden LE: Signaling pathways for PC12 cell differentiation: making the right connections. Science (New York, NY) 2002, 296(5573):1648-1649.

30. Fujita K, Lazarovici P, Guroff G: Regulation of the differentiation of PC12 pheochromocytoma cells. Environmental health perspectives 1989, 80:127-142.

31. Sysi-Aho M, Katajamaa M, Yetukuri $L$, Oresic M: Normalization method for metabolomics data using optimal selection of multiple internal standards. BMC bioinformatics 2007, 8:93.

32. Wu W, Dave N, Tseng GC, Richards T, Xing EP, Kaminski N: Comparison of normalization methods for CodeLink Bioarray data. BMC bioinformatics 2005, 6:309.

33. Ravni A, Vaudry D, Gerdin MJ, Eiden MV, Falluel-Morel A, Gonzalez BJ, Vaudry H, Eiden LE: A CAMP-dependent, protein kinase A-independent signaling pathway mediating neuritogenesis through Egr1 in PC12 cells. Molecular pharmacology 2008, 73(6):1688-1708.

34. Mark MD, Liu Y, Wong ST, Hinds TR, Storm DR: Stimulation of neurite outgrowth in PC12 cells by EGF and $\mathrm{KCl}$ depolarization: a $\mathrm{Ca}(2$ +)-independent phenomenon. The Journal of cell biology 1995, 130(3):701-710.

35. Pacary E, Petit E, Bernaudin M: Concomitant inhibition of prolyl hydroxylases and ROCK initiates differentiation of mesenchymal stem cells and PC12 towards the neuronal lineage. Biochemical and biophysical research communications 2008, 377(2):400-406.

36. Politis PK, Thomaidou D, Matsas R: Coordination of cell cycle exit and differentiation of neuronal progenitors. Cell cycle (Georgetown, Tex) 2008, 7(6):691-697.

37. Burkhalter J, Fiumelli H, Allaman I, Chatton JY, Martin JL: Brain-derived neurotrophic factor stimulates energy metabolism in developing cortical neurons. J Neurosci 2003, 23(23):8212-8220.

38. Salton SR: Neurotrophins, growth-factor-regulated genes and the control of energy balance. The Mount Sinai journal of medicine, New York 2003, 70(2):93-100.

39. da Silva JS, Dotti CG: Breaking the neuronal sphere: regulation of the actin cytoskeleton in neuritogenesis. Nature reviews 2002, 3(9):694-704.

40. Pak CW, Flynn KC, Bamburg JR: Actin-binding proteins take the reins in growth cones. Nature reviews 2008, 9(2):136-147.

41. Suzuki T, Higgins PJ, Crawford DR: Control selection for RNA quantitation. BioTechniques 2000, 29(2):332-337.

42. Silver N, Best S, Jiang J, Thein SL: Selection of housekeeping genes for gene expression studies in human reticulocytes using real-time PCR. BMC molecular biology 2006, 7:33. 
43. Thorrez L, Van Deun K, Tranchevent LC, Van Lommel L, Engelen K, Marchal K, Moreau Y, Van Mechelen I, Schuit F: Using ribosomal protein genes as reference: a tale of caution. PLOS ONE 2008, 3(3):e1854.

44. Lafontaine DL, Tollervey D: The function and synthesis of ribosomes. Nat Rev Mol Cell Biol 2001, 2(7):514-520.

45. Kenmochi N, Kawaguchi T, Rozen S, Davis E, Goodman N, Hudson TJ, Tanaka T, Page DC: A map of 75 human ribosomal protein genes. Genome research 1998, 8(5):509-523.

46. Robledo S, Idol RA, Crimmins DL, Ladenson JH, Mason PJ, Bessler M: The role of human ribosomal proteins in the maturation of rRNA and ribosome production. RNA (New York, NY) 2008, 14(9):1918-1929.

47. Faliks $D$, Meyuhas $\mathrm{O}$ : Coordinate regulation of ribosomal protein mRNA level in regenerating rat liver. Study with the corresponding mouse cloned cDNAs. Nucleic acids research 1982, 10(3):789-801.

48. Kirn-Safran CB, Dayal S, Martin-DeLeon PA, Carson DD: Cloning, expression, and chromosome mapping of the murine Hip/Rpl29 gene. Genomics 2000, 68(2):210-219.

49. Wanzel M, Russ AC, Kleine-Kohlbrecher D, Colombo E, Pelicci PG, Eilers M: A ribosomal protein L23-nucleophosmin circuit coordinates Miz1 function with cell growth. Nat Cell Biol 2008, 10:1051-1061.

50. He H, Sun Y: Ribosomal protein S27L is a direct p53 target that regulates apoptosis. Oncogene 2007, 26(19):2707-2716.

51. Jeon YJ, Kim IK, Hong SH, Nan H, Kim HJ, Lee HJ, Masuda ES, Meyuhas O, Oh BH, Jung YK: Ribosomal protein $\mathrm{S} 6$ is a selective mediator of TRAILapoptotic signaling. Oncogene 2008, 27(31):4344-4352.

52. Lee NH, Weinstock KG, Kirkness EF, Earle-Hughes JA, Fuldner RA, Marmaros S, Glodek A, Gocayne JD, Adams MD, Kerlavage AR, et al: Comparative expressed-sequence-tag analysis of differential gene expression profiles in PC-12 cells before and after nerve growth factor treatment. Proceedings of the National Academy of Sciences of the United States of America 1995, 92(18):8303-8307.

53. Angelastro JM, Klimaschewski L, Tang S, Vitolo OV, Weissman TA, Donlin LT, Shelanski ML, Greene LA: Identification of diverse nerve growth factorregulated genes by serial analysis of gene expression (SAGE) profiling. Proceedings of the National Academy of Sciences of the United States of America 2000, 97(19):10424-10429.

54. Too HP: Real time PCR quantification of GFRalpha-2 alternatively spliced isoforms in murine brain and peripheral tissues. Brain Res Mol Brain Res 2003, 114(2):146-153.

55. Bustin SA, Benes V, Garson JA, Hellemans J, Huggett J, Kubista M, Mueller R, Nolan T, Pfaffl MW, Shipley GL, et al: The MIQE guidelines: minimum information for publication of quantitative real-time PCR experiments. Clinical chemistry 2009, 55(4):611-622.

doi:10.1186/1471-2164-11-75

Cite this article as: Zhou et al:: Normalization with genes encoding ribosomal proteins but not GAPDH provides an accurate quantification of gene expressions in neuronal differentiation of $\mathrm{PC} 12$ cells. BMC Genomics 2010 11:75.

\section{Submit your next manuscript to BioMed Central and take full advantage of:}

- Convenient online submission

- Thorough peer review

- No space constraints or color figure charges

- Immediate publication on acceptance

- Inclusion in PubMed, CAS, Scopus and Google Scholar

- Research which is freely available for redistribution

Submit your manuscript at www.biomedcentral.com/submit
Biomed Central 